\title{
The place of omega-3 and omega- 6 acids in supplementary treatment of inflammatory joint diseases
}

\author{
Brygida Kwiatkowska, Maria Maślińska \\ Early Arthritis Clinic, National Institute of Geriatrics, Rheumatology and Rehabilitation, Warsaw, Poland
}

\begin{abstract}
Eating habits have been analysed for years as a factor influencing the development of autoimmune diseases and susceptibility to infections. On the basis of research, observational studies and metaanalyses, special attention was paid to omega- 3 and omega- 6 acids. The purpose of the review is to show the importance of omega- 3 and omega- 6 acids as important ingredients in the healthy diet and as factors protecting against the development of the most common inflammatory rheumatic diseases. The influence of these omega- 3 and -6 acids on the course of rheumatic diseases and arguments for their use as complementary therapy are also presented.
\end{abstract}

Key words: rheumatic diseases, omega-3, omega- 6 acids.

\section{Introduction}

Inflammatory joint diseases are enumerated among chronic autoimmune diseases, which mostly affect young people and result in changes within multiple organs and lead to limited mobility.

Both genetic factors (not modifiable) and environmental factors (some of which can be modified) may have an influence on the development of these diseases. Current scientific research focuses on testing genetic factors increasing the risk related to the development of the disease in people who are still healthy. In the future this could possibly allow implementation of prophylactic therapy and analysis of environmental factors, as well as enabling an attempt to modify them in order to reduce the likelihood of being affected by the disease or modify the course of the existing inflammatory joint disease.

Smoking is one of the environmental factors posing a recognised and considerable influence on the development or the course of inflammatory joint diseases such as rheumatoid arthritis (RA) and psoriatic arthritis (PSA). Active smokers are at 20\% higher risk of development of RA than non-smokers [1].

As far as PSA is concerned, smoking increases the risk of being affected by this disease in the general population, whereas the majority of trials indicate a reduced risk of suffering from PsA within the population of patients with psoriasis. This phenomenon is known as the "smoking paradox" [2].

Body weight is yet another environmental factor influencing the development and the course of inflammatory joint diseases. Psoriatic arthritis reveals clear correlations between obesity and the risk related to the development of the disease and its course. What concerns patients with psoriasis, the frequency associated with the prevalence of PSA increases along with the growing body mass index (BMI) [3]. What is more, obesity in patients suffering from PsA also results in worsened efficiency of administered treatment, even one based on biological medications [4, 5]. Positive correlations between the activity of the disease and obesity have also been observed in patients suffering from RA [6].

Physical activity is indicated as the next environmental factor which could possibly influence the development of RA. It has been reported that physical activity (> 20 minutes a day/hour a week) such as working at home, walking, strolling, riding a bicycle, swimming, doing aerobics, jogging and playing tennis can reduce the risk of developing RA $[7,8]$.

Many reports indicate that there is a causal link between infections and the development of inflammatory rheumatism. 
There is currently a discussion about the importance of infection caused by Porphyromonas gingivalis, resulting in protein citrullination and hence leading to chronic periodontal disease as a triggering factor of RA development. De Smith et al. [9] studied seropositive patients with arthralgia and seropositivity of antibodies against $P$. gingivalis and concluded that these antibodies do not predict RA development, but another study by Johansson et al. [10] showed that antibodies against. P. gingivalis were increased in RA patients and may emerge years before the disease onset.

Nevertheless, infections with this particular bacterium are more frequently observed in patients with RA and they impact the activity of the disease in the DAS28 assessment [11].

Recent studies have shown that a change in human microflora, especially the intestinal microbiota, plays a crucial role in the pathogenesis underlying many inflammatory rheumatic diseases including RA, spondyloarthropathies (SpA), systemic lupus erythematosus (SLE), systemic sclerosis (SS), antiphospholipid syndrome (APS) and Sjögren's syndrome (SS) [12].

The human microbiota is filled not only with commensal microorganisms, but also with clearly pathogenic ones. There are 3 main mechanisms related to how these microorganisms act in rheumatic diseases.

Firstly, commensal microorganisms may deplete nutrients and thereby limit the growth of new bacteria, which impacts the immune defence mechanisms [13].

Secondly, microorganisms may activate the immune system directly by the fact that their products cross the intestinal lamina propria and adhere to the specific receptors on the surface of the antigen-presenting cells, such as dendritic cells and macrophages. This, in turn, activates the $T$ cells and initiates the production of pro-inflammatory cytokines such as IL-1, IL-6, IL-17, TNF- $\alpha$, as well as stimulating the production of various antibodies [14].

Thirdly, overgrowth of certain intestinal microorganisms may cause the excessive production of metabolites - short-chain fatty acids (SCFAs) such as acetate, butyrate or propionate, which contribute to the development of comorbidities. These metabolites may lead to obesity by signalling pathways coupled with the G protein (GPR41, GPR43), which then impacts not only the course, but also the treatment of diseases such as RA or PsA [15].

\section{Diet and its influence on the development and course of rheumatic diseases}

A number of recent scientific studies have indicated that diet plays a considerable role in interacting with the microbiome, exerting an influence on its functions and the health status. During the last several years we could observe a drastic shift in the diet of highly developed countries.

The diet exhibits a radical drop in the consumption of fibre, along with a significant increase in the consumption of simple sugars, fats and proteins of animal origin, as well as highly processed foodstuff, which substantially impacts the microbiome, induces pro-inflammatory reactions and increases the risk of auto-immune diseases [16, 17]. Increased consumption of sodium chloride is another factor contributing to the activation of pro-inflammatory reactions through the Th17 pathway [18].

Diet is a factor that can have an influence on both the development and the course of RA. Hu et al. [19] in 2017 published results of a study based on the analysis of 93,392 women aged between 25 and 42 years old and 76,597 women aged between 30 and 55 years old and found an association with lower RA risk (HRQ4 vs. Q1: 0.67; 95\% Cl 0.51 to 0.88; $p$ trend: 0.002) in the younger group. Such an association is particularly important for seropositive women in this study group [19].

That is why diet should become an integral part of prevention and treatment of inflammatory rheumatic diseases (IRDs). Dietary regimens recommended in IRD are based on the elements of the Mediterranean diet, which covers a balanced composition of food based on vegetables, olives, fish, fruit, nuts, cereal grains and legumes, along with moderate consumption of eggs, lean meat, dairy and alcohol [20-23].

Exceptional substances in the Mediterranean diet currently draw attention due to the diversity of their health promoting properties. These substances include n-3 (omega-3) and n-6 (omega-6) polyunsaturated fatty acids, as well as n-9 (omega-9) monounsaturated fatty acids.

\section{General characteristics of omega-3 and omega- 6 acids, and their occurrence in nature}

Omega-3 and omega- 6 acids are polyunsaturated acids, diverse in terms of the location of their first double bond from the side of the methyl group (at the third or sixth carbon atom located farthest from the carboxylic acid group, labelled as omega).

The group of omega-3 acids includes alpha-linolenic acid (ALA), eicosapentaenoic acid (EPA) and docosahexaenoic (DHA), whereas the group of omega- 6 acids covers linoleic acid (LA), gamma-linolenic acid (GLA) and arachidonic acid (AA).

Omega-3 and omega-6 polyunsaturated fatty acids are not synthesized in the human organism and they 


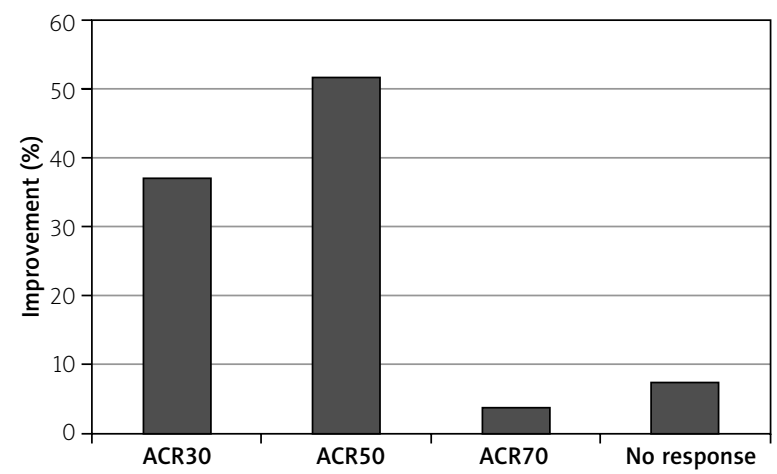

Fig. 1. Improvement of the paediatric ACR response in patients suffering from JIA after 12 weeks of using supplementation with omega-3 acids [33].

have to be provided with food being the source of these acids. Oily marine fish such as salmon, mackerel, tuna, herring and seafood represent the major and the richest source of EPA and DHA, whereas ALA is of plant origin and can be found in rapeseed, walnut oil, almonds and leafy greens.

Omega-6 acids can be obtained from soy, corn, sunflower oil, egg yolk, oily fish and seafood. The most considerable amounts of GLA acid, enumerated among omega- 6 acids, can be obtained from borage, evening primrose, true hemp, blueweed and blackcurrant.

The transformation of omega- 3 and omega- 6 acids is observed in the endoplasmic reticulum, where they are transformed into long chain polyunsaturated fatty acids (LC-PUFA), which have essential biological activity. What concerns these transformations, these acids compete with one another for the same enzymes.

Due to the above, the prevalence of omega- 6 (LA) acids in the diet inhibits the synthesis of EPA and DHA, whereas it increases the production of arachidonic acid (AA), which can impair the physiological balance of the whole system. Eicosanoids, such as prostaglandin, thromboxane or leukotrienes, originate from omega-3 and omega- 6 acids. Eicosanoids originating from omega- 6 acids reveal strong proinflammatory, prothrombotic and atherogenic activity, while omega-3 acids (EPA and DHA) are known for their anti-inflammatory, antithrombotic, vasodilating and anti-atherogenic properties [24-26].

Nonetheless, it seems favourable to combine omega-3 acids with the GLA in the diet or in the implemented supplementation. The addition of GLA to omega-3 acids results in decelerating the production of leukotrienes and preventing the accumulation of arachidonic acid, which leads to an anti-inflammatory effect $[27,28]$. Omega-3 acids also exert an influence on lipid management by lowering the concentration of triglycerides [29].
It is therefore crucial to maintain suitable proportions between omega- 6 and omega- 3 acids consumed within our everyday diet in products containing these acids or through their supplementation. It has been shown that the ratio between omega- 6 and omega- 3 acids is optimal when the proportion ranges between $2.5: 1$ and $5: 1$ (omega-6 : omega-3) [30].

\section{The significance of diet supplementation with omega- 3 and omega- 6 acids in management of patients with inflammatory rheumatic disorders}

\section{Juvenile idiopathic arthritis}

Juvenile idiopathic arthritis (JIA) of the joints is the most common chronic, inflammatory autoimmune joint disease in developmental age, with its onset observed under 16 years of age. During the active phase of the disease we can observe increased levels of TNF- $\alpha$, INF- $\gamma$, IL- 6 and IL-1 $\beta$, both in the serum and in the synovial fluid in patients suffering from JIA [31].

A study focusing on the Polish population of patients with JIA conventionally treated with anti-inflammatory drugs and immunosuppressants such as non-steroidal anti-inflammatory drugs (NSAID), glucocorticosteroids (GCS), methotrexate (MTX), chloroquine (HQ), hydroxychloroquine (HCQ) and sulfasalazine (SF) showed that the concentration of omega- 3 and omega- 6 acids had a negative correlation with the number of involved joints, value of the erythrocyte sedimentation rate (ESR) and the concentration of CRP, along with a positive correlation with the number of blood platelets [32].

The benefit of supplementation with omega-3 acids has been confirmed by a 12-week trial, during which patients with JIA were taking omega-3 acids for 12 weeks in a dose of $2 \mathrm{~g} /$ day [33]. The effectiveness of this therapy was evaluated on the basis of the Juvenile Arthritis Disease Activity Score on 27 joints (JADAS-27) and the Paediatric American College of Rheumatology (ACR) response criteria.

The improvement within the functionality was assessed based on the Childhood Health Assessment Questionnaire (CHAQ). After 12 weeks of supplementing patients with omega-3 acids, the researchers observed a statistically significant reduction in the number of ailing and swollen joints, the JADAS-27 index and CHAQ. Improvement within the Paediatric ACR response criteria was observed in $92.59 \%$ of patients (Fig. 1).

Before introducing the omega-3 supplementation all patients were taking diclofenac sodium, where $74.07 \%$ of patients were taking the dose of $150 \mathrm{mg} /$ day, 18.52\% of patients were taking $100 \mathrm{mg} /$ day, and $2 \%$ were taking 
$50 \mathrm{mg} /$ day. After 2 weeks of supplementation $88.888 \%$ of patients stopped taking NSAID (diclofenac sodium), $2 \%$ continued therapy, but in the dose of $50 \mathrm{mg} / \mathrm{day}$, whereas 1 patient was taking $100 \mathrm{mg} /$ day.

In patients suffering from JIA, who were taking supplementation with omega-3 acids, it was also observed that the concentration of pro-inflammatory cytokines, namely IL-1 and TNF- $\alpha$, in blood serum had decreased [33]. Therefore, supplementation with omega-3 acids should constitute a supplementary therapy.

\section{Ankylosing spondylitis}

Ankylosing spondylitis (AS) is enumerated among the axial spondyloarthropathies, in case of which the onset of the disease is observed before the age of 40 . The disease is associated with chronic pain and fatigue, where the chronic inflammatory process leads to progressive limitation of agility and worsens the quality of life.

Currently there are a few trials focusing on the significance related to supplementation with omega-3 and omega- 6 acids in supplementary treatment for this disease. Nevertheless, the analysis of recent publications indicates that nowadays there are no properly conducted studies which could explicitly confirm that there is a diet having a considerable influence on the course of this disease [34].

However, it has been shown that there is a positive correlation between the activity of this disease and the concentration of arachidonic acid in plasma phospholipids and adipose tissue phospholipids in the assessment based on the Bath Ankylosing Spondylitis Disease Activity Index (BASDAI) [35].

All the same, a randomized trial evaluating the impact of omega-3 acid supplementation applied in 2 groups of patients, taking either a small dose (1.95 g of omega-3/ day) or a large dose (4.55 g of omega-3/day), revealed a statistically significant reduction in the activity of the disease according to the BASDAI assessment in the group taking a large dose of omega-3 acids [36]. However, due to the small number of patients (only 18 patients, out of 48 in both groups, finished the study) these results require further.

\section{Psoriatic arthritis}

Psoriatic arthritis (PSA) is a chronic inflammatory joint disease mainly affecting the facet joints when it occurs in patients under 40 years of age, and affecting peripheral joints in people over 40 years of age.

The metabolic syndrome may occur in as many as $50 \%$ of patients suffering from PA who observed the onset of the disease before reaching 40 years of age. Dyslipidaemia is reported in $74.5 \%$ of patients, arterial hypertension in $61.8 \%$, obesity in $52.5 \%$ and type 2 diabetes in $30.9 \%$ of patients $[37,38]$. All these comorbidities increase the risk of cardiovascular complications, not to mention the risk of dying.

It has been shown that the implementation of omega-3 acids in a dose equalling 3 g/day for 24 weeks in patients with PA improves the autonomic and hemodynamic functions of the heart by impacting the value of arterial pressure, the central blood pressure and cardiac function, and reducing the heart rate variability (HRV) and pulse wave velocity (PWV) [39]. This has a direct effect on reducing the risk of cardiovascular complications and the sudden death resulting from these events.

On the other hand, another trial focusing on $145 \mathrm{pa}$ tients suffering from PA who were given omega-3 acids in a dose of $3 \mathrm{~g} /$ day or were given $3 \mathrm{~g}$ of olive oil showed that patients taking omega-3 acids revealed reduced activity of the disease under the DAS28-CRP assessment, decreased number of painful joints (evaluation covers 68), improvement within the scope of the enthesitis index and skin coverage according to the Psoriasis Area and Severity Index (PASI).

Nonetheless, these differences were not statistically significant when compared with the control group. However, patients taking omega-3 acid supplements revealed a statistically significant reduction in taking NSAID and acetaminophen when compared with patients taking olive oil. Decreased concentration of leukotriene B4, which is produced by leukocytes during the inflammatory process, was the statistically significant change observed within the group treated with omega-3 acids [40], whereas significant consumption of omega- 6 acids and linolenic acid (LA) reduced the risk of developing PsA, and not psoriasis [41].

\section{Rheumatoid arthritis}

Rheumatoid arthritis (RA) is the most common IRD. This disease tends to affect more women than men, and the peak of its incidence is observed between the third and the fifth decade of life. Studies on the influence of environmental factors on both the course and the development of this disease have been conducted for many years. Diet is one of these factors. It has been documented that a balanced diet maintaining suitable proportions between omega- 3 and omega- 6 acids reduces the risk of developing this condition and improves the effectiveness of its treatment.

Currently there are many studies revealing considerable effectiveness of omega-3 supplementation in supplementary therapy of this disease.

One of the studies, conducted in Sweden, which enrolled 727 patients with preliminary RA, proved that according to EULAR a statistically significant more frequent 
positive response to the therapy based on DMARDs (disease-modifying antirheumatic drugs), mainly MTX, was observed in patients who followed a diet rich in omega-3 acids [42]. Among patients treated with MTX and following a diet rich in omega-3 acids, an increased value of liver enzymes was less frequently observed [42].

A double-blind, randomised study covering $60 \mathrm{pa}$ tients suffering from RA treated with standard DMARDs and taking 3 capsules with an overall dose equalling 1.8 $\mathrm{g}$ of EPA and $2.1 \mathrm{~g}$ of DHA for 3 months vs. patients taking placebo for 12 months revealed that the actively treated group showed clinical improvement within the following scope: shortened morning stiffness reduced from $128 \mathrm{mi}$ nutes to 40 minutes, reduction of painful joints from 21 to 5 , reduced number of swollen joints from 10 to 3 , as well as lowered ESR value from $39 \mathrm{~mm}$ after 1 hour to $16 \mathrm{~mm}$ after 1 hour. 10\% of patients reduced the dose of taken DMARDs, whereas 32\% stopped taking these medications completely [43].

Another double-blind, randomised trial covered 60 patients with RA, divided into 3 groups (20 patients in each group):

- group I - patients were taking 5 capsules containin $1 \mathrm{~g}$ of fish oil (300 mg of DHA, $200 \mathrm{mg}$ of EPA and $100 \mathrm{~m}$ of other omega-3 acids),

- group II - patients were taking 2 capsules of fish oil used in group I and 2 capsules containing $1300 \mathrm{mg}$ of evening primrose oil, including $949 \mathrm{mg}$ of LA and $117 \mathrm{mg}$ of GLA,

- group III - patients were undergoing standard therapy [44].

All patients were given the same doses of glucocorticosteroids, methotrexate and NSAID. After 12 weeks a statistically significant reduction in the activity of the disease according to DAS28 was observed in groups | ( $4.99 \pm 0.88$ vs. $3.91 \pm 0.8)$ and II $(4.76 \pm 0.85$ vs. $3.7 \pm 0.72)$ compared with group III (4.66 \pm 0.80 vs. $4.23 \pm 0.66)$, in which no supplementation had been implemented.

Statistically significant differences between groups I and II and group III were also observed in ESR and CRP values, as well as in the number of painful and swollen joints, along with the experienced pain according to the VAS scale [44].

The impact on the course and the effectiveness of the standard RA therapy has also been shown in a randomised study, where the analysis covered the correlation between the concentration of plasma phospholipids originating from omega-3 acids and the probability of remission or treatment failure.

The analysed group consisted of 139 patients suffering from RA for less than 12 months and previously not treated with DMARDs. All patients started a 3 DMARD therapy (SF 0.5 and then $1 \mathrm{~g} 2 \mathrm{x}$ day, HCQ $200 \mathrm{mg} 2 \mathrm{x}$ day and MTX $10 \mathrm{mg} /$ week). Methotrexate was increased up to the dose reaching $25 \mathrm{mg}$ per week only in patients who revealed continuous, significant activity of the disease. Patients were divided in a $2: 1$ ratio into a group taking large doses $(10 \mathrm{ml})$ of fish oil $(3.2 \mathrm{~g}$ EPA $+2.3 \mathrm{~g}$ DHA) and a group taking small doses of fish oil (0.21 g $\mathrm{EPA}+0.19 \mathrm{~g}$ DHA).

A year-long observation revealed remission according to ACR and DAS28 in $61 \%$ of patients taking large doses of omega-3 vs. 33\% of patients taking small doses of omega- 3 acids. An increase in concentration by 1 unit of EPA phospholipids (as a percent of the overall amount of omega-3 phospholipids) in blood serum increases the remission probability by about $12.2 \%$ [45].

Despite the fact that a significant number of trials indicating the advantages that omega-3 acid supplementation can have on the course of RA was based on a small group of patients, a conducted meta-analysis covering 20 available studies with 1288 patients suffering from RA confirmed that omega-3 acid supplementation has an undeniable positive influence on the duration of morning stiffness, grip strength, ESR values, the number of painful joints, pain experienced by the patient, agility of the patient according to $\mathrm{HAQ}$ and decreased leukotriene B4 concentration [46]. High concentration of omega- 6 LA in the erythrocyte membrane through its supplementation reduces the risk of developing RA in patients with the pre-clinical phase of RA (pre-RA) [47]. Patients with RA face an increased risk of cardiovascular diseases and death caused by these diseases. The above results from the overall maintained inflammation, which directly impacts the more rapidly developing atherosclerotic lesions.

A study conducted on a group of 60 patients with RA, divided into 3 groups, where patients in group I were taking 5 capsules containing $1 \mathrm{~g}$ of fish oil (1 capsule contained $300 \mathrm{mg}$ of DHA $+200 \mathrm{mg}$ of EPA and $100 \mathrm{mg}$ of other omega-3 acids), patients in group II were taking 2 capsules of fish oil and 1 capsule of evening primrose oil (949 mg of LA + 117 mg of GLA) for 3 months, whereas patients in group III continued the previously recommended therapy.

When compared to group III, both groups taking supplements revealed a statistically significant reduction of blood platelet aggregation assessed with the adenosine diphosphate (ADP) to thrombin receptor-activation peptide (TRAP) ratio, which reduces the risk of cardiovascular complications [48].

Based on the available analysis, it would be recommended to add omega-3 supplementation to the previously recommended RA treatment as a form of supplementary therapy, which could increase the effectiveness of the whole therapy and reduce the risk of cardiovascu- 
lar complications and the risk of death associated with this disease.

\section{Sjögren's syndrome}

Sjögren's syndrome is a chronic inflammatory auto-immune disease, which leads to inflammation and causes injury to exocrine glands (mainly lacrimal and salivary glands) along with simultaneous inflammatory lesions in multiple systems and organs. Sjögren's syndrome tends to affect women more frequently than men, and the peak of its incidence is observed between the fifth and the sixth decade of life.

The first randomised trials evaluating the effectiveness of implementing omega- 3 and omega- 6 supplementation in supplementary treatment of this disease have been conducted in the last several years. It has been shown that supplementing omega-3 acids for 3 months leads to a statistically significantly increased amount of produced saliva (without stimulation) when compared to a placebo group [49], whilst the use of omega-3 acids in combination with omega- 6 acids caused decreased activity of the disease evaluated with the ESSDAI index (EULAR Sjögren's syndrome disease activity index), as well as improvement of ocular lesions assessed with the Sicca Ocular Staining Score index, and it also increased the amount of produced saliva [50].

\section{Gout}

Gout is a type of crystal deposition disease, where monosodium urate crystals (MSU) are deposited in tissues causing acute and chronic inflammation and various kinds of clinical symptoms. Metabolic syndrome is diagnosed in $62.8 \%$ of patients suffering from gout.

Treatment for gout usually includes pharmacological treatment and implementation of a diet aiming to reduce the purine content in consumed meals. Recent studies have shown that, similarly to other rheumatic diseases, the Mediterranean diet, namely a diet rich in polyunsaturated fatty acids, influences the level of uric acid and decreases the frequency of gout attacks [51].

The recently observed increasing number of patients suffering from gout results from the growing number of obese people consuming excessive amounts of meat, as well as from rising consumption of fructose [52].

In relation with the above, there have been a number of trials evaluating the effectiveness of diet and omega-3 supplementation in gout. The analysis covered 724 patients suffering from gout, which was diagnosed based on ACR criteria dating back to 1977 , with $22 \%$ of patients following an omega-3 diet or a diet rich in these acids.

It has been shown that a high omega-3: omega-6 ratio leads to reduced risk of subsequent gout attacks, while a neutral omega-3: omega-6 ratio has no impact on the course of the gout, and finally a low omega-3: omega- 6 ratio increases the risk of its relapse. This changes the traditional approach towards dietary treatment in the case of gout, which recently included recommendations to limit the intake of oily fish, and this means fish rich in omega-3 fatty acids. Nonetheless, it is worth remembering the recommendation to limit the overall purine intake [53].

\section{Conclusions}

Supplementation of omega-3 acids impacts the course of many inflammatory rheumatic diseases by decreasing their activity, reducing the pain, as well as reducing the risk of cardiovascular complications. However, there are indications that GLA should be added to the omega-3 supplementation, as it intensifies the anti-inflammatory activity of omega-3 acids. Therefore supplementation with omega-3 acids combined with GLA should become a part of complex therapies used for treating the following diseases: JA, spondyloarthropathies (AS, PSA), RA, Sjögren's syndrome and gout.

Brygida Kwiatkowska has conducted lectures during conferences, conventions and business meetings in cooperation with the pharmaceutical companies Pfizer, MSD, Sandoz, Roche, Eli Lilly, UCB, AbbVie, Novartis.

Maria Maślinska has conducted lectures for the pharmaceutical companies Pfizer, Sandoz, Roche, UCB, AbbVie, Novartis.

This study was not funded.

\section{References}

1. Sparks JA, Chen CY, Hiraki LT, et al. Contributions of familial rheumatoid arthritis or lupus and environmental factors to risk of rheumatoid arthritis in women: a prospective cohort study. Arthritis Care Res (Hoboken) 2014; 66: 1438-1446, DOI: 10.1002/acr.22366.

2. Nguyen UDT, Zhang Y, Lu N, et al. Smoking paradox in the development of psoriatic arthritis among patients with psoriasis: a population-based study. Ann Rheum Dis 2018; 77: 119-123, DOI: 10.1136/annrheumdis-2017-211625.

3. Love TJ, Zhu Y, Zhang Y, et al. Obesity and the risk of psoriatic arthritis: a population-based study. Ann Rheum Dis 2012; 71: 1273-1277, DOI: 10.1136/annrheumdis-2012-201299.

4. Højgaard P, Glintborg B, Kristensen LE, et al. The influence of obesity on response to tumor necrosis factor-alpha inhibitors in psoriatic arthritis: results from the DANBIO and ICEBIO registries. Rheumatology (Oxford) 2016; 55: 2191-2199, DOI: 10.1093/rheumatology/kew326.

5. di Minno MN, Peluso R, lervolino S, et al. Obesity and the prediction of minimal disease activity: a prospective study in pso- 
riatic arthritis. Arthritis Care Res (Hoboken) 2013; 65: 141-147, DOI: 10.1002/acr.21711.

6. Nikiphorou E, Norton S, Young A, et al. The association of obesity with disease activity, functional ability and quality of life in early rheumatoid arthritis: data from the Early Rheumatoid Arthritis Study/Early Rheumatoid Arthritis Network UK prospective cohorts. Rheumatology (Oxford) 2018; 57: 1194 1202, DOI: 10.1093/rheumatology/key066.

7. Di Giuseppe D, Bottai M, Askling J, Wolk A. Physical activity and risk of rheumatoid arthritis in women: a population-based prospective study. Arthritis Res Ther 2015; 17: 40, DOI: 10.1186/ s13075-015-0560-2.

8. Liu X, Tedeschi SK, Lu B, et al. Long-Term Physical Activity and Subsequent Risk for Rheumatoid Arthritis Among Women: A Prospective Cohort Study. Arthritis Rheumatol 2019; 71 1460-1471, DOI: 10.1002/art.40899.

9. De Smith M, van de Stadt LA, Janssen KM, et al. Antibodies against Porphyromonas gingivalis in seropositive arthralgia patients do not predict development of rheumatoid arthritis. Ann Rheum Dis 2014; 73: 1277-1279, DOI: 10.1136/annrheumdis2013-204594.

10. Johansson L, Sherina N, Kharlamova N, et al. Concentration of antibodies against Porphyromonas gingivalis is increased before the onset of symptoms of rheumatoid arthritis, [This article has been corrected] Arthritis Res Ther 2016; 4;18: 257, DOI: 10.1186/s13075-016-1164-1.

11. Ceccarelli F, Orrú G, Pilloni A, et al. Porphyromonas gingivalis in the tongue biofilm is associated with clinical outcome in rheumatoid arthritis patients. Clin Exp Immunol 2018; 194: 244-252, DOI: 10.1111/cei.13184.

12. De Luca F, Shoenfeld Y. The microbiome in autoimmune diseases. Clin Exp Immunol 2019; 195: 74-85, DOI: 10.1111/ cei.13158.

13. Abdollahi-Roodsaz S, Abramson SB, Scher JU. The metabolic role of the gut microbiota in health and rheumatic diseases: mechanisms and interventions. Nat Rev Rheumatol 2016; 12: 446-455, DOI: 10.1038/nrrheum.2016.68.

14. Van de Wiele, Van Praet JT, Marzorati M, et al. How the microbiota shapes rheumatic diseases. Nat Rev Rheumatol 2016; 12: 398-411, DOI: 10.1038/nrrheum.2016.85.

15.Zhong D, Wu C, Zeng, Wang Q. The role of gut microbiota in the pathogenesis of rheumatic diseases. Clin Rheumatol 2018; 37: 25-34, DOI: 10.1007/s10067-017-3821-4.

16. Derrien M, Veiga P. Rethinking Diet to Aid Human-Microbe Symbiosis. Trends Microbiol 2017; 25: 100-112, DOI: 10.1016/j.tim.2016.09.011.

17.Zinöcker M, Lindseth IA. The Western Diet - Microbiome-Host Interaction and Its Role in Metabolic Disease. Nutrients 2018; 10: e 365, DOI: 10.3390/nu10030365.

18. Sharif K, Amital H, Shoenfeld Y. The role of dietary sodium in autoimmune diseases: The salty truth. Autoimmun Rev 2018; 17: 1069-1073, DOI: 10.1016/j.autrev.2018.05.007.

19. Hu Y, Sparks JA, Malspeis S, et al. Long-term dietary quality and risk of developing rheumatoid arthritis in women. Ann Rheum Dis 2017; 76: 1357-1364, DOI: 10.1136/annrheumdis2016-210431.

20. Oliviero F, Spinella P, Fiocco U, et al. How the Mediterranean diet and some of its components modulate inflammatory pathways in arthritis. Swiss Med Wkly 2015; 145: w14190, DOI: 10.4414/smw.2015.14190.

21. Jin Z, Xiang C, Cai Q et al. Alcohol consumption as a preventive factor for developing rheumatoid arthritis: a dose-response meta-analysis of prospective studies. Ann Rheum Dis 2014; 73: 1962-1967, DOI: 10.1136/annrheumdis-2013-203323.

22. Hedström AK, Hössjer O, Klareskog L, Alfredsson L. Interplay between alcohol, smoking and HLA genes in RA aetiology. RMD Open 2019; 5: e000893, DOI: 10.1136/rmdopen-2019-000893.

23. Paolino $S$, Pacini $G$, Patanè $M$, et al. Interactions between microbiota, diet/nutrients and immune/inflammatory response in rheumatic diseases: focus on rheumatoid arthritis. Reumatologia 2019; 57: 151-157, DOI: 10.5114/reum.2019.86425.

24. Thies F, Garry JM, Yaqoob P, et al. Association of $n-3$ polyunsaturated fatty acids with stability of atherosclerotic plaques: a randomized controlled trial. Lancet 2003; 363: 477-485, DOI: 10.1016/S0140-6736(03)12468-3.

25. Kris-Etherton PM, Harris WS, Appel LJ, et al. Fish consumption, fish oil, omega- 3 fatty acids, and cardiovascular disease. Arterioscler Tromb Vasc Biol 2003; 23: e20-30, DOI: 10.1161/01. atv.0000038493.65177.94.

26. Sekikawa A, Doyle MF, Kuller LH. Recent findings of long-chain n-3 polyunsaturated fatty acids (LCn-3 PUFAs) a atherosclerosis and coronary heart disease (CHD) contrasting studies in Western countries to Japan. Trends Cardiovasc Med 2015; 25: 717-723, DOI: 10.1016/j.tcm.2015.03.001.

27. Barham JB, Edens MB, Fonteh AN, et al. Addition of eicosapentaenoic acid to gamma-linolenic acid-supplemented diets prevnets serum arachidonic acid accumulation in humans. J Nutr 2000; 130: 1925-1931, DOI: 10.1093/jn/130.8.1925.

28. Sergeant S, Rahbar E, Chilton FH. Gamma-linolenic acid, Dihommo-gamma linolenic, Eicosanoids and Inflammatory Process. Eur J Pharmacol 2016; 785: 77-86, DOI: 10.1016/j. ejphar.2016.04.020.

29. Harris WS, Bulchandani D. Why do omega-3 fatty acids lower serum triglycerides? Curr Opin Lipidol 2006; 17: 387-393, DOI: 10.1097/01.mol.0000236363.63840.16.

30. Simopoulos AP. The importance of the omega-6/omega-3 fatty acid ratio in cardiovascular disease and other chronic diseases. Exp Biol Med (Maywood) 2008; 233: 674-688, DOI: 10.3181/0711-MR-311.

31. Spîrchez M, Samaşca G, lancu M, et al. Relation of interleukin-6, TNF-alpha and interleukin-1 alpha with disease activity and severity in juvenile idiopathic arthritis. Clin Lab 2012; 58: 253-260.

32.Gorczyca D, Postępski J, Czajkowska A, et al. The profile of polyunsaturated fatty acids in juvenile idiopathic arthritis and association with disease activity. Clin Rheumatol 2017; 36: 1269-1279, DOI: 10.1007/s10067-017-3586-9.

33. Gheita T, Kamel S, Helmy N, et al. Omega-3 fatty acids in juvenile idiopathic arthritis: effect on cytokines (IL-1 and TNF- $\alpha$ ), disease activity and response criteria. Clin Rheumatol 2012; 31: 363-366, DOI: 10.1007/s10067-011-1848-5.

34. Macfarlane TV, Abbood HM, Pathan E, et al. Relationship between diet and ankylosing spondylitis: A systemic review. Eur J Rheumatol 2018; 5: 45-52, DOI: 10.5152/eurjrheum. 2017.16103. 
35. Sundström B, Johansson G, Kokkonen $\mathrm{H}$, et al. Plasma phospholipid fatty acid content is related to disease activity in ankylosing spondylitis. J Rheumatol 2012; 39: 327-333, DOI: 10.3899/jrheum.110575.

36. Sundström B, Stålnace K, Hagfors L, et al. Supplementation of omega-3 fatty acids in patients with ankylosing spondylitis. Scand J Rheumatol 2006; 35: 359-362, DOI: 10.1080/03009740600844357.

37. Caso F, Del Puente A, Oliviero F, et al. Metabolic syndrome in psoriatic arthritis: the interplay with cutaneous involvement. Evidence from literature and recent cross-sectional study. Clin Rheumatol 2018; 37: 579-586, DOI: 10.1007/s10067-0173975-0.

38. Souza CS, de Castro CCS, Carneiro FRO, et al. Metabolic syndrome and psoriatic arthritis among patients with psoriasis vulgaris: Quality of life and prevalence. J Dermatol 2019; 46: 3-10, DOI: 10.1111/1346-8138.14706.

39. Kristensen S, Schmidt EB, Schlemmer A, et al. The effect of marine $n-3$ polyunsaturated fatty acids on cardiac autonomic and hemodynamic function in patients with psoriatic arthritis: a randomized, double-blind, placebo-controlled trial. Lipids Health and Dis 2016; 15: 216, DOI: 10.1186/s12944-016 0382-5.

40. Kristensen S, Schmidt EB, Schlemmer A, et al. Beneficial effect of $n-3$ polyunsaturated fatty acids on inflammation and analgesic use in psoriatic arthritis: a randomized, double blind, placebo-controlled trial. Scand J Rheumatol 2018; 47: 27-36, DOI: 10.1080/03009742.2017.1287304.

41. Park MK, Li W, Paek SY, et al. Consumption of polyunsaturated fatty acids and risk of incident psoriasis and psoriatic arthritis from the Nurses' Health Study II. Br J Dermatol 2017; 177 302-306, DOI: 10.1111/bjd.15059.

42. Lourdudoss C, Wolk A, Nise L, et al. Are dietary vitamin D, omega-3 fatty acids and folate associated with treatment results in patients with early rheumatoid arthritis? Data from a Swedish population-based prospective study. BMJ Open 2017; 7: e016154, DOI: 10.1136/bmjopen-2017-016154.

43. Rajaei E, Mowla K, Ghorbani A, et al. The effects of Omega-3 Fatty Acids in Patients With Active Rheumatoid Arthritis Receiving DMARDs Therapy: Double-Blind Randomized Controlled Trial. Glob J Health Sci 2015; 8: 18-25, DOI: 10.5539/ gjhs.v8n7p18.
44. Veselinovic M, Vasiljevic D, Vucic V, et al. Clinical Benefits of n-3 PUFA and $\gamma$-Linolenic Acid in Patients with Rheumatoid Arthritis. Nutrients 2017; 9, e325, DOI: 10.3390/nu9040325.

45. Proudman SM, Cleland LG, Metcalf RG, et al. Plasma $n-3$ fatty acids and clinical outcomes in recent-onset rheumatoid arthritis. Br J Nutr 2015; 114: 885-890, DOI: 10.1017/ S0007114515002718.

46. Gioxari A, Kaliora AC, Marantidou F, Panagiotakos DP. Intake of $\omega-3$ polyunsaturated fatty acids in patients with rheumatoid arthritis: A systemic review and meta-analysis. Nutrition 2018; 45: 114-124, DOI: 10.1016/j.nut.2017.06.023.

47. de Pablo P, Romaguera D, Fisk HL, et al. High erythrocyte levels of the n-6 polyunsaturated fatty acid linoleic acid are associated with lower risk of subsequent rheumatoid arthritis in southern European nested case-control study. Ann Rheum Dis 2018; 77: 981-987, DOI: 10.1136/annrheumdis-2017-212274.

48. Tomic-Smiljanic M, Vasiljevic D, Tomic-Lucic A, et al. Influence of different supplementation on platelet aggregation in patients with rheumatoid arthritis. Clin Rheumatol 2019; 38: 2443-2450, DOI: 10.1007/s10067-019-04569-3.

49. Papas A, Singh $M$, Singh $M$. The effect of a Unique Omega-3 Supplement on Dry Mouth and Dry Eye in Sjögren's Patients. IOVS 2007; 48: 377, DOI: 10.1186/ISRCTN10758297.

50. Hemandez-Molina G, Castrejón-Morales C, Granados-Portillo 0 , et al. Omega-3 and Omega- 6 Fatty Acids in Sjögren's syndrome: clinical implications and their association with inflammation. Ann Rheum Dis 2019; 78: 798, DOI: 10.1136/ annrheumdis-2019-eular.5646.

51.Stamostegiou J, Theodoris X, Ganachoriti V, et al. The role of the Mediterranean diet in hyperuricemia and gout. Mediterr J Rheumatol 2018; 29: 21-25.

52. Ayoub-Charette S, Lui Q, Khan TA, et al. Important food sources of fructose-containing sugars and incident gout: a systematic review and meta-analysis of prospective cohort studies. BMJ Open 2019; 9: e024171, DOI: 10.1136/bmjopen-2018-024171.

53.Zhang M, Zhang Y, Terkeltaub R, et al. Effect of Dietary and Supplemental Omega-3 Polyunsaturated Fatty Acids on Risk of Recurrent Gout Flares. Arthritis Rheumatol 2019; 71: 15801586, DOI: 10.1002/art.40896. 